\title{
Development of mono and multilayer antimicrobial food packaging materials for controlled release of potassium sorbate
}

\author{
Metin Uz, Sacide Alsoy Altınkaya* \\ Department of Chemical Engineering, Faculty of Engineering, Izmir Institute of Technology, 35430 Gülbahçe Köyü, Urla, Izmir, Turkey
}

\section{A R T I C L E I N F O}

\section{Article history:}

Received 8 October 2010

Received in revised form

10 April 2011

Accepted 4 May 2011

\section{Keywords:}

Antimicrobial food packaging

Controlled release

Cellulose acetate

Potassium sorbate

Drying induced crystallization

\begin{abstract}
A B S T R A C T
In this study, cellulose acetate (CA) based mono and multilayer films including potassium sorbate (Psb) as an antimicrobial agent were prepared using dry phase inversion technique. To achieve appropriate controlled release of Psb, the structure of the films was changed by manipulating the film preparation conditions. In particular, the initial casting composition, wet casting thickness and drying temperature were varied. Results indicate that Psb release rate decreased as the CA content in the casting solution, the wet casting thickness and the drying temperature for both mono and multilayer films were increased. Compared to the results for the monolayer films, a significant decrease of Psb release rate through the multilayer films was recorded. Drying-induced crystallization was observed in the monolayer films. As a consequence of this, a fast initial release of Psb, controlled by Fickian diffusion, was followed by a slower release controlled by dissolution of Psb crystals. In multilayer films, no crystals were detected in the structure and the release rate was regulated only by diffusion of Psb through the film. The results suggest that the films prepared in this study can be used as food packaging materials for achieving controlled and extended release of Psb.
\end{abstract}

(c) 2011 Elsevier Ltd. All rights reserved.

\section{Introduction}

Recently, the use of antimicrobial packaging has received great attention since it provides foods high quality, safety and long shelf life by reducing, inhibiting or retarding the growth of microorganisms (Appendini \& Hotchkiss, 2002; Choi et al., 2005). Traditionally, antimicrobial agents are directly mixed into the initial food formulations. Direct addition, however, may result in a decrease in the concentration of the antimicrobial agent on the food surface due to its diffusion into the interior parts of the food. Therefore, the minimum concentration required for the inhibition of the microbial growth may not be achieved and the antimicrobial compound cannot selectively target the food surface (Min \& Krochta, 2005). In addition, the neutralization of the added agent due to its possible complex interactions with the food components may occur (Appendini \& Hotchkiss, 2002). Moreover, the direct addition brings about the utilization of excessive amounts of the antimicrobial agent which may change the taste of the food. Antimicrobial packaging offers an alternative way to overcome these limitations.

\footnotetext{
* Corresponding author. Tel.: +90 232 7506658; fax: +90 2327506645 .

E-mail address: sacidealsoy@iyte.edu.tr (S.A. Altınkaya).
}

The most important desired property of the antimicrobial packaging materials is the controlled release of the antimicrobial agent from the film to the food surface. A rapid release causes fast consumption of the agent in a short period of time, after which the minimum concentration required for the inhibition of microbial growth is not maintained on the food surface. On the other hand, spoilage reactions on the food surface may start if the release rate of the antimicrobial agent from the film is too slow. Thus, the controlled release of the active agent for a long period of time is necessary to extend the shelf life of the packed food.

Studies on the development of antimicrobial food packaging films with controlled release properties are increasing. Different controlled release strategies were introduced which are mainly based on changing the structure of the films. Han and Floros (1998) tried to control the release of an active agent by using a three layered film structure. The first layer is the outer barrier layer, whose function is to prevent the migration of the agent to the environment, the second is a matrix layer containing the active agent, and the third controls the release of the active agent to the food. Buonocore, Conte, Corbo, Sinigaglia, and Del Nobile (2005) and Mastromatteo, Barbuzzi, Conte, and Del Nobile (2009) also developed multilayer films consisting of two external control layers and an inner layer containing the active agent. In another work of Buonocore's group, they tried to manipulate the release kinetics of 


\begin{tabular}{|c|c|}
\hline \multicolumn{2}{|c|}{ Nomenclature } \\
\hline \multicolumn{2}{|c|}{ List of symbols } \\
\hline$A_{f}$ & Area of the film $\left(\mathrm{cm}^{2}\right)$ \\
\hline $\mathrm{D}$ & $\begin{array}{l}\text { Effective diffusion coefficient of active agent in the } \\
\text { film }\left(\mathrm{cm}^{2} / \mathrm{sec}\right)\end{array}$ \\
\hline $\mathrm{k}$ & Crystal dissolution constant $\left(\min ^{-1}\right)$ \\
\hline $\mathrm{K}$ & Partition coefficient $\left(\mathrm{cm}^{3}\right.$ solution $/ \mathrm{cm}^{3}$ film $)$, \\
\hline $\mathrm{L}$ & Thickness of the film $(\mathrm{cm})$ \\
\hline $\mathrm{M}_{\text {Total }}$ & $\begin{array}{l}\text { Total amount of active agent desorbed from the film } \\
\text { at any time } \mathrm{t}(\mathrm{mg}) \text {, }\end{array}$ \\
\hline$M \infty$ & $\begin{array}{l}\text { Total amount of active agent desorbed from the film } \\
\text { at equilibrium (mg), }\end{array}$ \\
\hline $\mathrm{M}_{\text {Fickian }}$ & $\begin{array}{l}\text { Total amount of active agent desorbed from the film } \\
\text { by Fickian diffusion (mg), }\end{array}$ \\
\hline \multicolumn{2}{|c|}{$\begin{array}{l}\mathrm{M}_{\text {Dissolution }} \text { Total amount of active agent desorbed from the } \\
\text { film by crystal dissolution ( } \mathrm{mg} \text { ) }\end{array}$} \\
\hline & Time (min), \\
\hline & Volume of the solution $\left(\mathrm{cm}^{3}\right)$ \\
\hline
\end{tabular}

active compounds by changing the degree of cross-linking of the polymer matrix (Buonocore et al., 2003; Buonocore, Del Nobile, Panizza, Corbo, \& Nicolais, 2003; Buonocore et al., 2004). Ozdemir and Floros (2001) targeted controlling the release by changing the degree of plasticization in the polymer film. Iconomopoulou and Voyiatzis (2005) suggested changing the molecular orientation of the polymer chains as a tool for achieving the controlled release of the active compounds. Guzey and McClements (2006) proposed micro and nano-encapsulation of food ingredients as another alternative method to control the release rate of the active agent. They suggested that the release rate from small capsules directly added into the food can be controlled by changes in $\mathrm{pH}$, temperature or ionic strength of the medium. Han, Castell-Perez, and Moreira (2008) investigated the effect of electron beam irradiation on the release kinetics and they suggested that irradiation may serve as a controlling factor for the release of the active compound. Recently, Gemili, Yemenicioglu, and Altinkaya (2009; 2010) introduced the usage of porous asymmetric films for food packaging applications. They tried to control the release rates by changing the degree of asymmetry and porosity of the films.

Potassium sorbate, which is a potassium salt of the sorbic acid, is well-known for its potential antifungal activity and generally used in the preservation of cheese, dairy products and dough (ValenciaChamorro, Palou, Del Rio, \& Perez-Gago, 2008). Previously, different research groups added Psb in whey protein; k-carregeenan; cellulose triacetate; poly (maleic acid-co-olefine); polystyrene; polyethylene terephthalate; low density polyethylene and copolymer of vinylidene chloride (Choi et al., 2005; Han \& Floros, 1997; Fama, Flores, Gerschenson, \& Goyanes, 2006; Ozdemir \& Floros, 2003; Limjaroen, Ryser, Lockhart, \& Harte, 2003; Flores et al., 2007; Vartiainen, Skytta, Enqvist, \& Ahvenainen, 2003; Ye, Neetoo, \& Chen, 2008). These published results indicate quick desorption of the Psb from the film, inducing a rapid loss of its activity. Thus, it is necessary to control and delay the release of the Psb from the films by investigating new film preparation strategies. In this study, a dry phase inversion technique has been used to prepare cellulose acetate based mono and multilayer food packaging materials including potassium sorbate as an antimicrobial agent. In this technique, the polymer is dissolved in a mixture of a volatile solvent and a less volatile non-solvent. After casting the solution on a glass support, it is exposed to an air stream for drying. During drying, the homogeneous solution phase separates into polymerlean and polymer-rich phases as a result of the difference in the evaporation rate of the solvent and non-solvent. The polymer-rich phase forms the matrix of the film while the polymer-lean phase rich in solvent and non-solvent fills the pores. Consequently, when the solvent and non-solvent are completely removed from the film, porous structure is obtained. Previously, we have shown that the dry phase inversion technique can be used for controlling the release rate of lysozyme as well as natural antioxidants L-tyrosine and ascorbic acid from cellulose acetate (CA) films by changing the porosity and pore size of the films. The morphological changes in the films have been achieved by changing the CA content in the film forming solutions. In this work, the influences of not only the initial casting composition but also the drying temperature and wet casting thickness on the release rates have been investigated for both mono and multilayer films. The wet casting thickness corresponds to the thickness of the polymer solution immediately after casting on a glass support before drying. To illustrate the advantage of the dry phase inversion technique in delaying the desorption of Psb, the monolayer CA film has also been prepared by traditional solvent evaporation technique commonly used in the literature. The main intent of the current study is to demonstrate that changing the film preparation method or conditions causes differences in the structural features of the films, which leads to obtaining different release profiles and, hence, allows manipulation of the release rate of Psb from these films.

\section{Materials}

Cellulose acetate with a molecular weight of 50,000 and acetyl content of 39.8\% was obtained from Eastman (Kingsport, TN, USA). Acetone (99\%) and potassium sorbate were obtained from Merc (Darmstadt, Germany) and AppliChem, respectively.

\section{Methods}

\subsection{Preparation of monolayer and multilayer films by dry phase inversion technique}

To prepare the monolayer films, potassium sorbate (Psb) and cellulose acetate (CA) were dissolved in water and acetone, respectively. Then, the Psb/water solution was poured into the CA/ acetone solution undergoing stirring. The stirring of the mixture continued until all the Psb solution was added and dissolved into the CA solution. Once the homogeneous mixture was obtained, the solution was left standing for $24 \mathrm{~h}$ to eliminate bubbles. The solution was then cast on a glass support with the aid of an automatic film applicator (Sheen, Automatic film applicator-1133N, Kingston, England) at a speed of $100 \mathrm{~mm} / \mathrm{s}$. The wet thickness of the film was adjusted by a four-sided applicator with the gap size of 300 or 500 micron. Immediately after casting, the film was placed into an environmental chamber (Siemens, Simatic OP7, Massa Martana, Italy) and dried for $1 \mathrm{~h}$ at $25^{\circ} \mathrm{C}$ or $50{ }^{\circ} \mathrm{C}$ and $40 \%$ relative humidity. Then, the films were peeled off from the glass support and further dried in a vacuum oven at $100{ }^{\circ} \mathrm{C}$ for a period of $24 \mathrm{~h}$. The Psb concentration in the film forming solutions was kept constant at $2 \%$ $(\mathrm{w} / \mathrm{w})$ while the CA $(10 \%, 12.5 \%, 15 \%$ by weight), acetone and water concentrations were changed.

In the case of multilayer films, $15 \%(\mathrm{w} / \mathrm{w})$ CA was dissolved in acetone and cast on a glass support with the aid of an automatic film applicator. Following 2 min of drying at $25^{\circ} \mathrm{C}$ or $50{ }^{\circ} \mathrm{C}$ and $40 \%$ relative humidity, the second layer, prepared in the same way as the monolayer films as described above, was cast on top of the first one. Finally, after $8 \mathrm{~min}$ of drying, the third layer, again made of $15 \%$ $(w / w)$ CA dissolved in acetone, was cast onto the second layer. The 
Table 1

Types of the films prepared, their codes, the porosity and average pore sizes.

\begin{tabular}{|c|c|c|c|c|c|c|}
\hline Code of the Film & Number of Layers & $\begin{array}{l}\text { Wet Casting Thickness } \\
(\mu \mathrm{m})\end{array}$ & $\begin{array}{l}\text { Temperature } \\
\left({ }^{\circ} \mathrm{C}\right)\end{array}$ & $\mathrm{CA} / \mathrm{H}_{2} \mathrm{O}^{\mathrm{a}}$ & Porosity $^{b}$ & $\begin{array}{l}\text { Pore size } \\
(\mu \mathrm{m})\end{array}$ \\
\hline \multirow[t]{3}{*}{ CA1 } & Mono & 300 & 25 & $10 / 28$ & $47.53 \pm 0.69$ & $6.09 \pm 0.2$ \\
\hline & Mono & 300 & 25 & $12.5 / 25.5$ & $40.45 \pm 0.64$ & $2.69 \pm 0.04$ \\
\hline & Mono & 300 & 25 & $15 / 23$ & $32.75 \pm 0.81$ & $1.18 \pm 0.02$ \\
\hline \multirow[t]{3}{*}{ CA2 } & Mono & 500 & 25 & $10 / 28$ & $32.74 \pm 0.49$ & $2.70 \pm 0.03$ \\
\hline & Mono & 500 & 25 & $12.5 / 25.5$ & $30.20 \pm 0.6$ & $1.75 \pm 0.06$ \\
\hline & Mono & 500 & 25 & $15 / 23$ & $26.45 \pm 0.7$ & $1.10 \pm 0.08$ \\
\hline \multirow[t]{3}{*}{ CA3 } & Mono & 300 & 50 & $10 / 28$ & $25.89 \pm 0.33$ & $3.48 \pm 0.07$ \\
\hline & Mono & 300 & 50 & $12.5 / 25.5$ & $23.94 \pm 0.48$ & $1.51 \pm 0.06$ \\
\hline & Mono & 300 & 50 & $15 / 23$ & $20.97 \pm 0.34$ & $0.92 \pm 0.05$ \\
\hline \multirow[t]{3}{*}{ CA4 } & Multi & 300 & 25 & $10 / 28$ & $19.56 \pm 0.34$ & $3.01 \pm 0.09$ \\
\hline & Multi & 300 & 25 & $12.5 / 25.5$ & $14.49 \pm 0.4$ & $1.43 \pm 0.06$ \\
\hline & Multi & 300 & 25 & $15 / 23$ & $8.94 \pm 0.71$ & $0.90 \pm 0.14$ \\
\hline \multirow[t]{3}{*}{ CA5 } & Multi & 500 & 25 & $10 / 28$ & $11.74 \pm 0.34$ & $2.47 \pm 0.11$ \\
\hline & Multi & 500 & 25 & $12.5 / 25.5$ & $10.18 \pm 0.3$ & $1.32 \pm 0.09$ \\
\hline & Multi & 500 & 25 & $15 / 23$ & $7.26 \pm 0.34$ & $0.85 \pm 0.11$ \\
\hline \multirow[t]{3}{*}{ CA6 } & Multi & 300 & 50 & $10 / 28$ & $10.83 \pm 0.23$ & $1.51 \pm 0.14$ \\
\hline & Multi & 300 & 50 & $12.5 / 25.5$ & $9.56 \pm 0.16$ & $1.00 \pm 0.1$ \\
\hline & Multi & 300 & 50 & $15 / 23$ & $8.63 \pm 0.2$ & $0.69 \pm 0.09$ \\
\hline
\end{tabular}

a The ratio corresponds to weight percentages of CA and water. Weight percentages of Psb and acetone in the film forming solution are 2 and 60 , respectively.

b Corresponds to volume percentages of the pores.

films were then dried for $1 \mathrm{~h}$ at $25{ }^{\circ} \mathrm{C}$ or $50{ }^{\circ} \mathrm{C}$ and $40 \%$ relative humidity in the environmental chamber and, thereafter, placed into a vacuum oven at $100{ }^{\circ} \mathrm{C}$ and kept there for $24 \mathrm{~h}$. The codes of the mono and multilayer films and corresponding preparation conditions are listed in Table 1.

\subsection{Preparation of monolayer films by solvent evaporation technique}

In the solvent evaporation technique, water was not used and Psb (2 wt \%) was directly dispersed in acetone (88 wt \%). After that, CA (10 wt \%) was dissolved in the Psb/acetone solution. The stirring was continued until all the CA content was dissolved and uniform dispersion of Psb was observed. The films were then cast and dried by the same procedure explained in Section 3.1.

\subsection{Release tests}

As will be shown in Section 4.1, due to the asymmetric and porous structure of the films prepared by the dry phase inversion technique, the release tests were conducted for both the dense and porous sides of the films separately. For this purpose, circularly cut film samples (5 $\mathrm{cm}$ in diameter) were squeezed between two

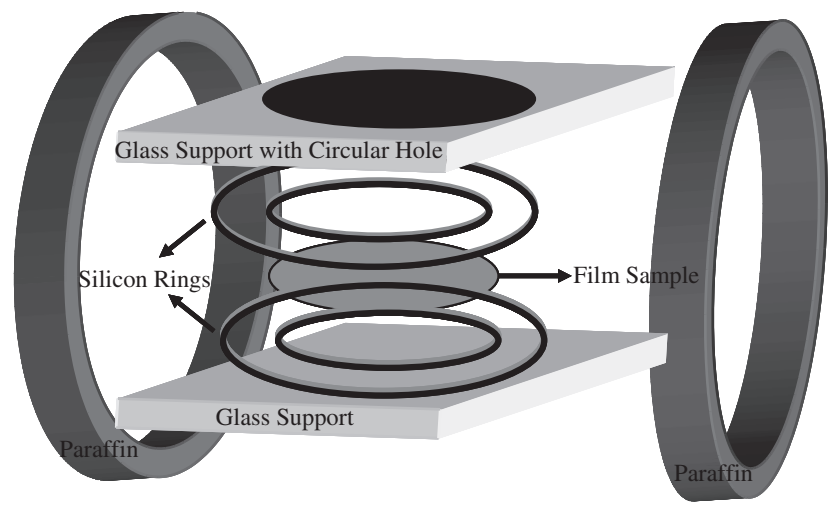

Fig. 1. Experimental set-up used in release tests of the films. circular silicon sealing rings as shown in Fig. 1. The surface to be tested (dense or porous) was sealed by a circular glass support containing a hole in the middle with a diameter of $5 \mathrm{~cm}$ to provide water contact and hence Psb release. The other surface of the film was also sealed by the glass support. However, it was surrounded with paraffin in order to prevent its contact with water and Psb release from this side. For release studies, the apparatus was placed into a glass Petri dish $(10 \mathrm{~cm}$ in diameter) containing $100 \mathrm{ml}$ distilled water with $\mathrm{pH} 7$ at $4{ }^{\circ} \mathrm{C}$ and stirred magnetically at $240 \mathrm{rpm}$ with a $2 \mathrm{~cm}$ long Teflon coated rod. The release of Psb into the medium was monitored by taking $100 \mu \mathrm{l}$ samples at different time periods. After diluting with $1 \mathrm{ml}$ distilled water, the absorbance of the samples was measured at $254 \mathrm{~nm}$ in UV spectrometer (Perkin Elmer Model No: Lambda 45). By using the calibration curve previously prepared for Psb, the concentration and the mass of the released Psb was calculated. During a release experiment, 3 samples, each with a volume of $100 \mu$, were taken from the release medium at approximately 20 different time intervals. Therefore, about $6 \mathrm{ml}$ was lost due to the measurements. The total volume of the release medium was measured at the end of the experiment and the volume loss due to evaporation was found to be negligible. The influence of the total volume loss was taken into consideration during the theoretical calculations and it was found that the results were not significantly affected by the decrease in the volume of the release medium.

\subsection{Morphological characterization of the films}

The morphology of the films was examined by scanning electron microscopy (SEM) on a Philips XL-30SFG model. For SEM analysis, samples were coated with gold palladium using a Magnetron Sputter Coating Instrument. Average pore size and porosity of the films were determined from image analysis of SEM micrographs. Morphological features were determined from image analysis of SEM micrographs and they are listed in Table 1.

\subsection{Determination of transport and thermodynamic parameters of potassium sorbate}

In cases where the release rate of Psb is controlled only by its diffusion through the film, Fick's second law was used as the main 


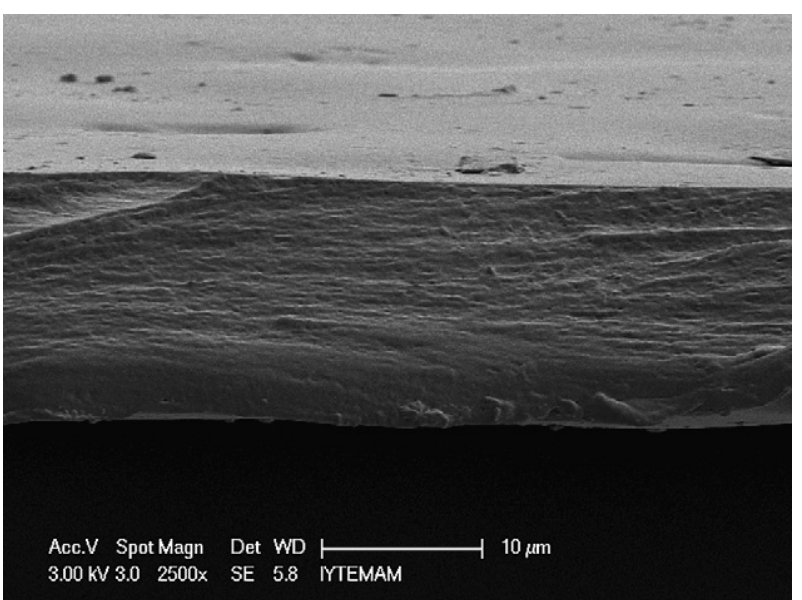

Fig. 2. SEM of the cross-section of the monolayer CA film prepared by the traditional solvent evaporation technique. The film was cast with $300 \mu \mathrm{m}$ wet casting thickness and dried at $25{ }^{\circ} \mathrm{C}$. The CA, acetone and Psb contents in the initial casting solution are $10 \mathrm{wt} \%, 2 \mathrm{wt} \%$ and $88 \%$, respectively. Magnification, $2500 \times$.

transport equation to describe the change in the concentration of Psb in the film with respect to time and position. To use this equation, it was assumed that there is no chemical reaction between Psb and the film, mass transfer is only by diffusion, the diffusivity of Psb is constant and the thickness of the film due to desorption of Psb does not change. A solution of Fick's second law along with one initial and two boundary conditions is presented in the classical book of Crank (1975). According to this solution, the total amount of active compound desorbed from the film at any time $t$, due to just Fickian diffusion, $M_{\text {Fickian }}$ is given by the following expression.

$$
M_{\text {Fickian }}=M_{\infty}\left[1-\sum_{n=1}^{\infty} \frac{2 \alpha(1+\alpha)}{1+\alpha+\alpha^{2} q n} \exp \left(-D q_{n}^{2} t / L^{2}\right)\right]
$$
where $M_{\infty}$ is the amount of Psb desorbed at equilibrium, $\alpha=\frac{V_{s o l}}{A_{f} L K}$
and the $q_{n}$ 's are the non-zero positive roots of

$\tan q_{n}=-\alpha q_{n}$

The partition coefficient, $K$, used in the definition of $\alpha$ is defined as the ratio of Psb concentration in the film to that in the solution at equilibrium and it was calculated from the difference in the equilibrium and the initial concentration of Psb measured in the solution assuming that potassium sorbate does not degrade during the assay.

Eq. (1) clearly indicates that in cases where the release is controlled only by Fickian diffusion, the diffusivity of Psb in the film is the main transport parameter and it was determined by minimizing the difference between the experimental data and the model predictions from Eq. (1).

As will be shown in Section 4.2, deviation from one stage Fickian sorption was observed due to dissolution of crystals formed during film preparation. In these cases, referred to as "two stage" sorption, the sorption curve is initially Fickian until it starts to level off. Instead of reaching the equilibrium level which is typical for Fickian sorption, the curve is extended through a non-Fickian part.

Berens and Hopfenberg (1978) have proposed a theory describing the features of this two stage sorption. The theory assumes that overall sorption is described by two phenomenologically independent contributions: a diffusion part, $M_{\text {Fickian, }}$ governed by Fick's law and a structural part resulting from polymer relaxation. The total weight loss/gain at any time $t$ is then expressed as a linear superposition of these contributions. Based on the similarity between the shape of the two stage sorption curves observed in this study due to crystal dissolution and those reported in the literature mainly due to polymer relaxation, we have adopted the theory proposed by Berens and Hopfenberg (1978). According to this theory, the total weight loss, $M_{t o t a l}$, is defined as follows;
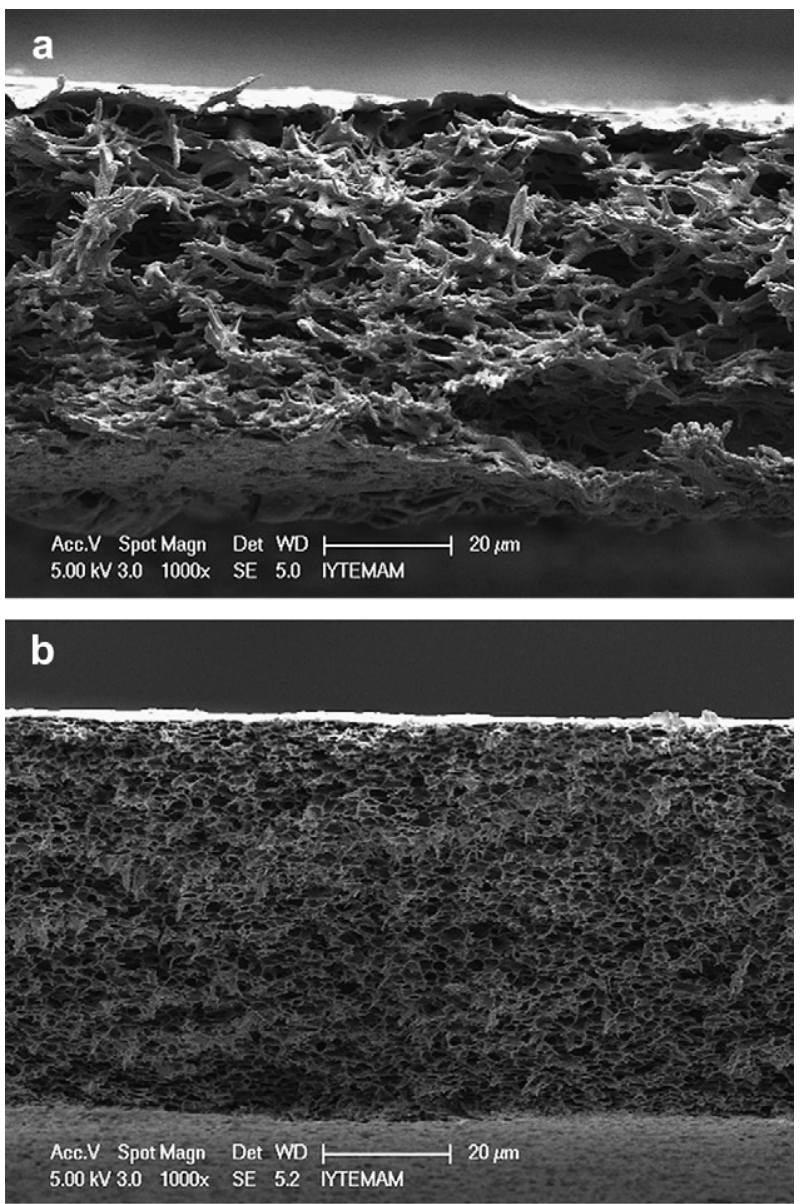

C

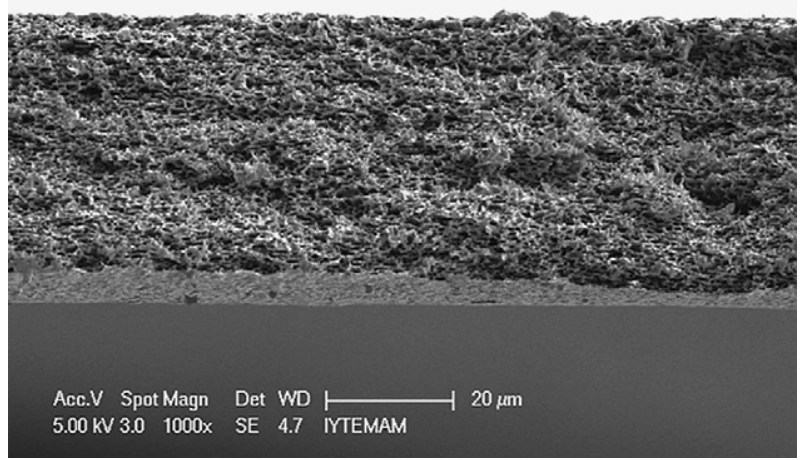

Fig. 3. SEM of the cross-sections of the monolayer CA1 films cast with $300 \mu \mathrm{m}$ wet casting thickness and dried at $25^{\circ} \mathrm{C}$. CA weight percentages in film forming solutions are a) 10 b) 12.5 c) 15 . Magnification, $1000 \times$. Upper sides of the films are the dense surfaces, while, bottom sides are porous surfaces. 

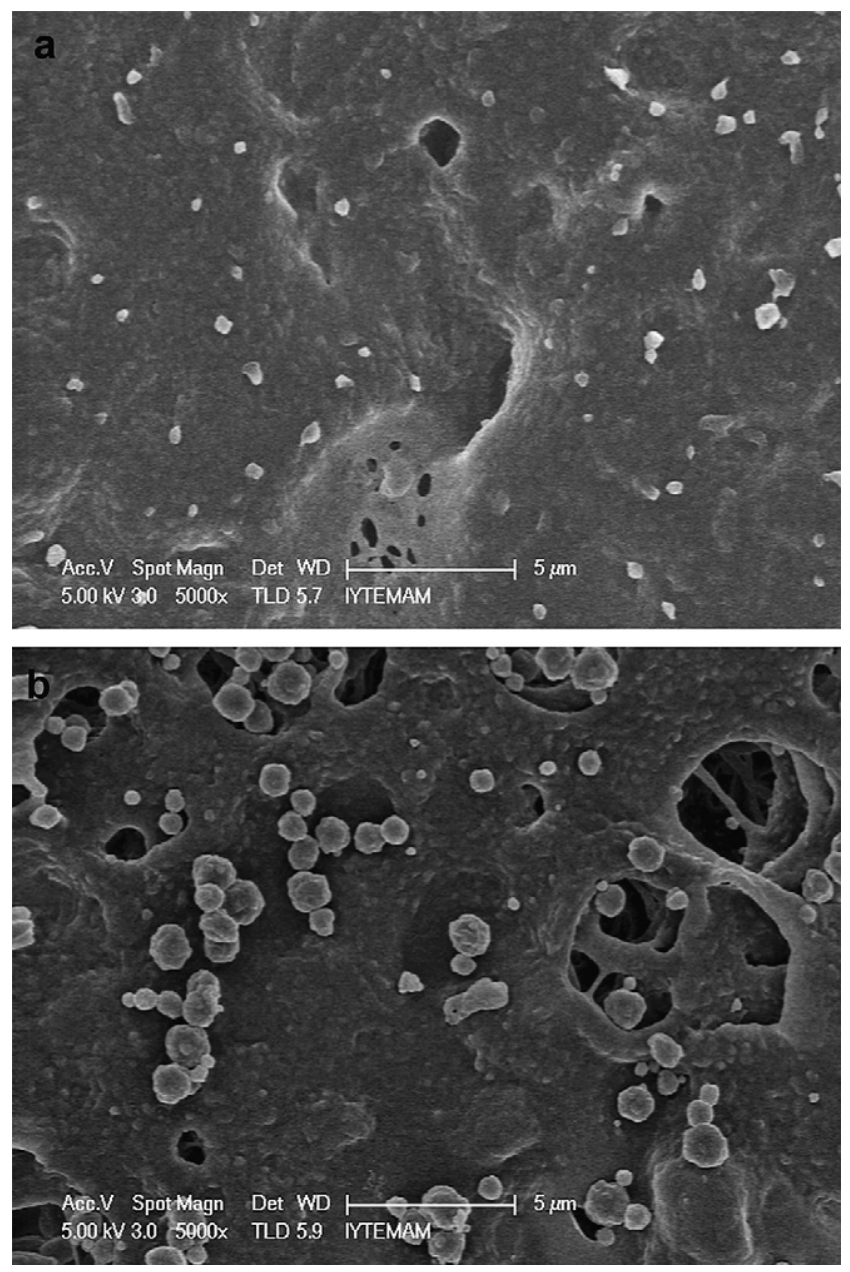

Fig. 4. SEM pictures of the crystals on the a) porous b) dense surfaces of CA1 film cast with $300 \mu \mathrm{m}$ thickness and dried at $25{ }^{\circ} \mathrm{C}$. The CA content in the initial casting solution is $10 \mathrm{wt} \%$. Magnification, $5000 \times$.

$M_{\text {Total }}(t)=M_{\text {Fickian }}(t)+M_{\text {Dissolution }}(t)$

where the weight loss due to dissolution of crystals is described by the following expression.

$M_{\text {Dissolution }}=M_{\infty}[1-\exp (-k t)]$

\subsection{Statistical analysis}

One of the goals of this study was to determine how the measured data on Psb transport, solubility, and crystal formation in mono and multilayer CA films were affected by a change in the experimental conditions. These experimental parameters included the film preparation conditions (CA content, wet casting thickness, and drying temperature) and whether Psb transport was through the porous or dense side of the film. To aid in this effort, an analysis of variance (ANOVA) was performed on the reported results, which were an average of three individual measures. During the analysis, only the experimental parameter of interest was changed while all others were kept constant. The difference in a particular measured property for Psb when comparing experiments was considered statistically insignificant when $p>0.05$ as determined by Tukey's protected least significant difference test.

\section{Results and discussion}

\subsection{Morphology of the films}

In this study, the dry phase inversion technique was used to prepare films as an alternative to conventional solvent evaporation technique. The SEM pictures shown in Figs. 2 and 3 indicate that the conventional solvent evaporation technique created a dense structure with no pores in it, while a porous structure has been obtained with the dry phase inversion technique. As can be inferred from Fig. 3, the monolayer films prepared by the phase inversion technique are not only porous but they are also asymmetric since the polymer concentration is not uniform throughout the cross section of the film. It can be seen that the porosity of the film decreases in the region near to the top surface, in fact, a very thin dense skin layer is observed at that surface, therefore, this surface will be denoted as dense surface while bottom side of the films will be denoted as porous surface. The SEM pictures of both dense and porous surfaces of the monolayer films indicated the presence of potassium sorbate crystals (Fig. $4 a$ and $b$ ). When water is removed from the film during drying in the vacuum oven, potassium sorbate molecules penetrate into the pores left by the water molecules. This raises supersaturation in the pores and, hence, induces the crystal growth. In the case of multilayer films, the rate of heat and mass transfer is lower due to existence of inner and outer layers. Thus, it can be expected that at any time during drying, moisture content in these films is higher than that in the monolayer films. Consequently, potassium sorbate does not reach concentration levels above its saturation limit to initiate crystallization and, as a result, no crystal formation was observed on the surfaces of the multilayer films.

The porosity and average pore size of the films were changed by varying the initial casting composition, wet casting thickness and drying temperature during the film preparation step. The results in Table 1 and in Fig. 3a through 3c clearly indicate that the increase in the polymer concentration from $10 \mathrm{wt} \%$ to $15 \mathrm{wt} \%$ or the increases in the wet casting thickness and drying temperature led to a statistically significant decrease $(p<0.05)$ in the pore size and porosity of the film. In addition, as the structure becomes denser, the size and amount of crystals decreased, which indicates that the crystal formation depends on pore size and porosity of the monolayer films.

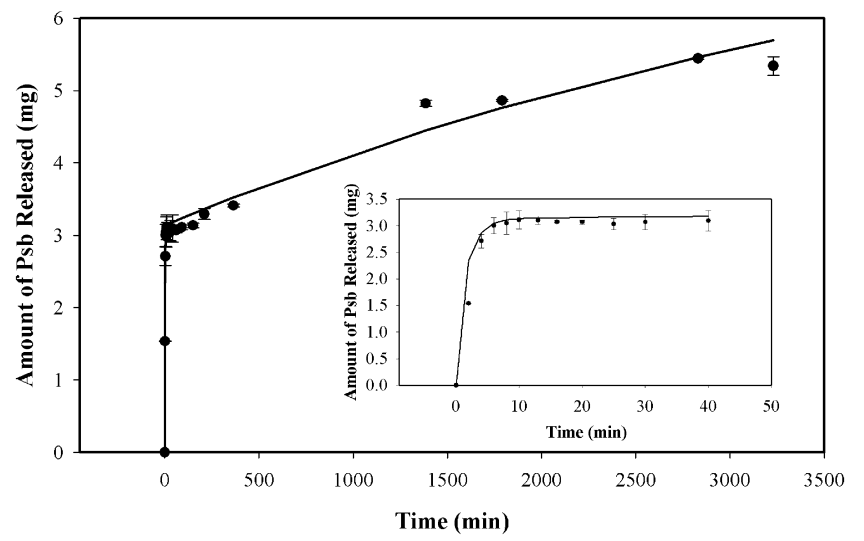

Fig. 5. The release profile of Psb obtained with the dense surface of the monolayer CA3 film cast with $300 \mu \mathrm{m}$ thickness and dried at $50{ }^{\circ} \mathrm{C}$. CA weight percentage in film forming solutions is $10 \mathrm{wt} \%$. (๑) and (-) represent experimental data and model predictions, respectively. 


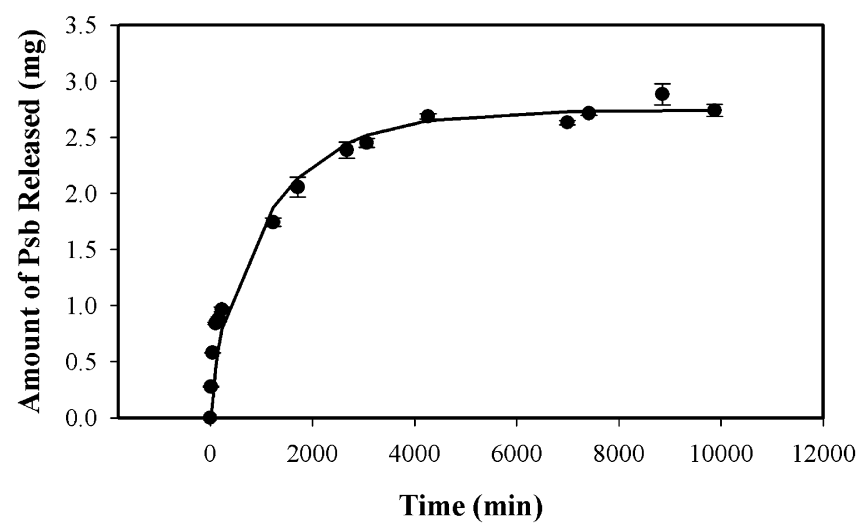

Fig. 6. The release profile of Psb obtained with the dense surface of the multilayer CA6 film cast with $300 \mu \mathrm{m}$ thickness and dried at $50{ }^{\circ} \mathrm{C}$. CA weight percentage in film forming solutions is $10 \mathrm{wt} \%$. (•) and (-) represent experimental data and model predictions, respectively.

\subsection{Release rates of Psb through the films}

The release profiles of the films prepared with the two different numbers of layers (monolayer and multilayer) and the three different polymer concentrations (10 wt \%, $12.5 \mathrm{wt} \%$ and $15 \mathrm{wt} \%$ ), cast with two different thicknesses $(300 \mu \mathrm{m}$ and $500 \mu \mathrm{m})$ and dried at two different temperatures $\left(25^{\circ} \mathrm{C}\right.$ and $\left.50^{\circ} \mathrm{C}\right)$ were measured. This corresponds to a total of 18 different films as shown in Table 1. Due to the asymmetric structure of the films, both dense and porous surfaces were used for the release studies. In the case of monolayer films, two stage sorption behavior was observed for each film composition. Fig. 5 illustrates that the initial release characteristics followed a Fickian behavior due to the release of uniformly distributed dissolved Psb particles. At longer times, the release rate was controlled by dissolution of Psb crystals. On the other hand, the release rate of Psb from all the multilayer films was controlled by Fickian diffusion and, hence, one stage sorption was observed, as illustrated in Fig. 6. The release characteristics for the dense monolayer film prepared by the traditional solvent evaporation technique (Fig. 7) also followed one stage sorption behavior since no crystal formation was observed in the structure, just as in the case of the multilayer films. The initial release rates from the

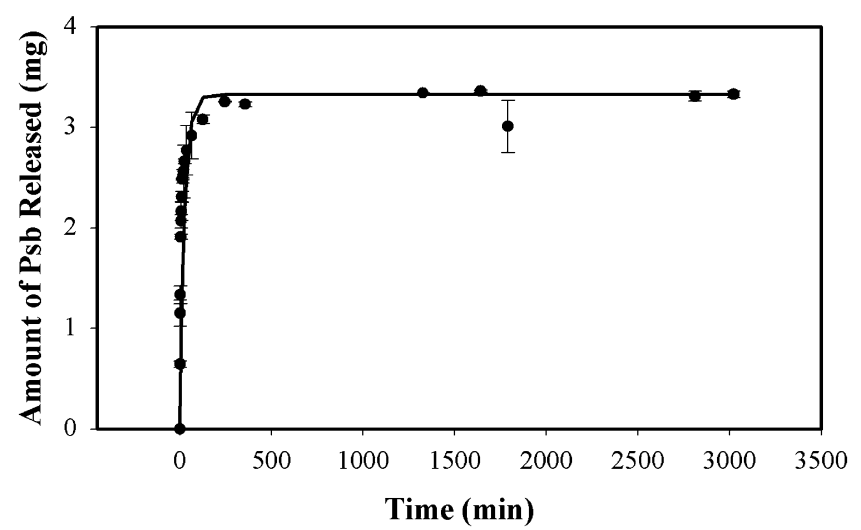

Fig. 7. The release profile of Psb obtained with the dense monolayer cellulose acetate film prepared by the traditional solvent evaporation technique. The wet casting thickness and the drying temperature are $300 \mu \mathrm{m}$ and $25^{\circ} \mathrm{C}$, respectively. The CA, acetone and Psb contents in the initial casting solution are $10 \mathrm{wt} \%, 2 \mathrm{wt} \%$ and $88 \%$, respectively. (-) and (-) represent experimental data and model predictions, respectively. monolayer films prepared by the traditional solvent evaporation and the dry phase inversion techniques were found to be similar. Therefore, the diffusion coefficients of Psb through these films are close to each other. On the other hand, the presence of crystals in the porous films prepared by the dry phase inversion technique extended the release of Psb and, as a result, equilibrium was reached in a much longer period of time. This clearly indicates the advantage of the dry phase inversion technique in comparison to the traditional solvent evaporation technique for extending the release of Psb by inducing crystal formation in the structure.

The diffusivities of Psb and the crystal dissolution constants were determined using the release profiles, and they are listed in Table 2. The results indicate that the diffusion coefficient of Psb decreased with the increase in the CA content in the films, the casting thickness and the drying temperature. This is due to a decrease in the porosity and pore size of the films, as confirmed by the results in Table 1 . Compared with the monolayer films, the diffusivities of Psb through the multilayer films were found to be two orders of magnitude lower due to the increased mass transfer resistance. The statistical analysis of the data for the diffusion coefficients, partition coefficients and the dissolution constants are shown in Table 2. In this table, the same letter in a row indicates a statistically insignificant difference $(p>0.05)$ in the coefficient determined using porous and dense surfaces of the films. On the other hand, the same letter in a column means that the coefficient/ constant does not change significantly $(p>0.05)$ when corresponding film preparation condition (i.e., the weight percentage of $\mathrm{CA} / \mathrm{H}_{2} \mathrm{O}$, the casting thickness and the drying temperature) was changed. The results indicate that the casting composition and thickness as well as the drying temperature of the solution in almost all cases have a significant effect on the diffusivities $(p<0.05)$ for both monolayer and multilayer films. In addition, the diffusivities of Psb obtained with the porous surfaces of all the films were found higher $(p<0.05)$ than those determined by using the dense surfaces.

The increases in the CA content and wet casting thickness during the film preparation caused a statistically significant difference $(p<0.05)$ in the crystal dissolution constant of the Psb in almost all cases. On the other hand, the drying temperature did not change the dissolution constants significantly $(p>0.05)$ especially when the porous surfaces of the films were used. The highest dissolution constants were obtained with the most porous film structures, which allow fast penetration of water into the film and thus dissolution of crystals at a fast rate. It is noted that, in most cases, the crystals present on the dense surfaces of the films dissolve at a faster rate than those which exist on the porous surfaces due to their smaller sizes. This indicates that the magnitude of crystal dissolution constant depends not only on the rate of water penetration to the crystal sites but also on the size of the crystals. All other conditions being the same, the differences in the crystal dissolution constants of the Psb obtained with the porous and dense surfaces of the films were found to be statistically significant $(\mathrm{p}<0.05)$.

The changes in the composition, the casting thickness and the drying temperature during the film preparation caused statistically significant differences ( $p<0.05)$, in almost all cases, in the partition coefficients of Psb in both monolayer and multilayer films, as shown in Table 2. With increased casting thickness and drying temperature, the amount of Psb immobilized in the multilayer films increased while, in most cases, an opposite trend was observed for the monolayer films. This indicates that in multilayer films Psb has a higher affinity to the denser structures. In the case of monolayer films, the amount of Psb retained in the film depends not only on the porosity but also on the presence of undissolved crystals in the structure. Due to this fact, the partition coefficients of Psb in the 
Table 2

Diffusion, partition coefficient and crystal dissolution constant of Psb obtained with the CA films prepared under different conditions.

\begin{tabular}{|c|c|c|c|c|c|c|c|}
\hline \multirow[t]{2}{*}{ Film Code } & \multirow[t]{2}{*}{$\mathrm{CA} / \mathrm{H}_{2} \mathrm{O}^{\mathrm{c}}$} & \multicolumn{2}{|l|}{ Diffusion Coefficient $\left(\mathrm{cm}^{2} / \mathrm{sec}\right)$} & \multicolumn{2}{|c|}{$\begin{array}{l}\text { Partition Coefficient }\left(\mathrm{cm}^{3}\right. \\
\left.\text { solution } / \mathrm{cm}^{3} \text { film }\right)\end{array}$} & \multicolumn{2}{|l|}{ Dissolution Constant $\left(\right.$ minute $^{-1}$ ) } \\
\hline & & Porous Surface $^{*}$ & Dense Surface $^{*}$ & $\begin{array}{l}\text { Porous } \\
\text { Surface* }\end{array}$ & $\begin{array}{l}\text { Dense } \\
\text { Surface* }\end{array}$ & $\begin{array}{l}\text { Porous } \\
\text { Surface }\end{array}$ & Dense Surface $^{*}$ \\
\hline CA1 $300 \mu \mathrm{m}^{\mathrm{a}}$ & $10 / 28$ & $9.65 \times 10^{-8} \pm 2.35 \times 10^{-10}$ & $4.23 \times 10^{-8} \pm 1.06 \times 10^{-10}$ & $426 \pm 1.9$ & $474 \pm 1.22$ & $2.03 \times 10^{-4} \pm 3.77 \times 10^{-6 \mathrm{~h}, \mathrm{n}}$ & $4.47 \times 10^{-4} \pm 4.52 \times 10^{-5 \mathrm{k}}$ \\
\hline \multirow{2}{*}{$25^{\circ} \mathrm{C}^{\mathrm{b}}$} & $12.5 / 25.5$ & $3.07 \times 10^{-8} \pm 4.8 \times 10^{-10}$ & $2.33 \times 10^{-8} \pm 2.36 \times 10^{-9}$ & $577 \pm 5$ & $613 \pm 1.74^{\mathrm{g}}$ & $2.03 \times 10^{-4} \pm 2.4 \times 10^{-6 \mathrm{i}, \mathrm{n}}$ & $2.34 \times 10^{-4} \pm 2.4 \times 10^{-5}$ \\
\hline & $15 / 23$ & $1.72 \times 10^{-8} \pm 1.76 \times 10^{-10}$ & $1.52 \times 10^{-8} \pm 3.08 \times 10^{-10}$ & $664 \pm 3.52$ & $824 \pm 1.2$ & $1.29 \times 10^{-4} \pm 6.36 \times 10^{-6 j}$ & $1.65 \times 10^{-4} \pm 3.54 \times 10^{-61}$ \\
\hline CA2 $500 \mu \mathrm{m}$ & $10 / 28$ & $3.3 \times 10^{-8} \pm 2.1 \times 10^{-9}$ & $3.08 \times 10^{-8} \pm 5.9 \times 10^{-10}$ & $399 \pm 3.22$ & $360 \pm 3.8$ & $1.02 \times 10^{-4} \pm 8.5 \times 10^{-6} \circ$ & $4.37 \times 10^{-4} \pm 2.6 \times 10^{-5 \mathrm{k}}$ \\
\hline \multirow[t]{2}{*}{$25^{\circ} \mathrm{C}$} & $12.5 / 25.5$ & $2.35 \times 10^{-8} \pm 1.1 \times 10^{-9}$ & $1.33 \times 10^{-8} \pm 4.13 \times 10^{-10}$ & $423 \pm 3.43$ & $435 \pm 3$ & $1.03 \times 10^{-4} \pm 9 \times 10^{-6 \circ}$ & $1.88 \times 10^{-4} \pm 2.69 \times 10^{-5}$ \\
\hline & $15 / 23$ & $1.52 \times 10^{-8} \pm 1.88 \times 10^{-10}$ & $8.68 \times 10^{-9} \pm 9.43 \times 10^{-11}$ & $585 \pm 1.63$ & $590 \pm 3.1$ & $1.32 \times 10^{-4} \pm 7.7 \times 10^{-6}$ & $0.9 \times 10^{-4} \pm 1.9 \times 10^{-6}$ \\
\hline CA3 $300 \mu \mathrm{m}$ & $10 / 28$ & $7.35 \times 10^{-8} \pm 7.87 \times 10^{-10}$ & $3.25 \times 10^{-8} \pm 8.82 \times 10^{-10}$ & $389 \pm 8.39$ & $545 \pm 9.84$ & $2.11 \times 10^{-4} \pm 1.71 \times 10^{-5} \mathrm{h,m}$ & $2.08 \times 10^{-4} \pm 2.14 \times 10^{-5 m}$ \\
\hline \multirow[t]{2}{*}{$50^{\circ} \mathrm{C}$} & $12.5 / 25.5$ & $2.52 \times 10^{-8} \pm 8.29 \times 10^{-10}$ & $1.62 \times 10^{-8} \pm 3.81 \times 10^{-10}$ & $493 \pm 2.55$ & $612 \pm 4.1^{\mathrm{g}}$ & $2.05 \times 10^{-4} \pm 9 \times 10^{-5 i}$ & $3.85 \times 10^{-4} \pm 7.85 \times 10^{-5}$ \\
\hline & $15 / 23$ & $1.62 \times 10^{-8} \pm 2.45 \times 10^{-10}$ & $1.35 \times 10^{-8} \pm 8.8 \times 10^{-10}$ & $833 \pm 6.9$ & $974 \pm 2.93$ & $1.21 \times 10^{-4} \pm 2 \times 10^{-5 j}$ & $2.38 \times 10^{-4} \pm 8 \times 10^{-51}$ \\
\hline CA4 $300 \mu \mathrm{m}$ & $10 / 28$ & $1.42 \times 10^{-9} \pm 1.92 \times 10^{-10} \mathrm{e}$ & $9.9 \times 10^{-10} \pm 1.2 \times 10^{-11}$ & $296 \pm 13.36$ & $320 \pm 8.28$ & - & - \\
\hline \multirow[t]{2}{*}{$25^{\circ} \mathrm{C}$} & $12.5 / 25.5$ & $9.02 \times 10^{-10} \pm 3.53 \times 10^{-12}$ & $6.18 \times 10^{-10} \pm 6.9 \times 10^{-12}$ & $323 \pm 12.36$ & $403 \pm 3.95$ & - & - \\
\hline & $15 / 23$ & $5.65 \times 10^{-10} \pm 1.29 \times 10^{-12}$ & $8.18 \times 10^{-10} \pm 4.71 \times 10^{-12}$ & $399 \pm 3.12$ & $530 \pm 5.27$ & - & - \\
\hline CA5 $500 \mu \mathrm{m}$ & $10 / 28$ & $9.18 \times 10^{-10} \pm 2.47 \times 10^{-12}$ & $4.18 \times 10^{-10} \pm 1.36 \times 10^{-12}$ & $422 \pm 5.57$ & $510 \pm 14.4$ & - & - \\
\hline \multirow[t]{2}{*}{$25^{\circ} \mathrm{C}$} & $12.5 / 25.5$ & $4.85 \times 10^{-10} \pm 1.19 \times 10^{-12}$ & $2.68 \times 10^{-10} \pm 1.18 \times 10^{-13}$ & $511 \pm 2.13$ & $486 \pm 1$ & - & _- \\
\hline & $15 / 23$ & $3.02 \times 10^{-} 10 \pm 2.47 \times 10^{-12}$ & $1.85 \times 10^{-10} \pm 1.31 \times 10^{-12}$ & $525 \pm 2.69^{f}$ & $523 \pm 5.8^{\mathrm{f}}$ & _- & _- \\
\hline CA6 $300 \mu \mathrm{m}$ & $10 / 28$ & $1.41 \times 10^{-9} \pm 1.29 \times 10^{-11} \mathrm{e}$ & $3.25 \times 10^{-10} \pm 2.1 \times 10^{-11}$ & $315 \pm 3.4$ & $416 \pm 1.7$ & _- & _- \\
\hline \multirow[t]{2}{*}{$50^{\circ} \mathrm{C}$} & $12.5 / 25.5$ & $5.68 \times 10^{-10} \pm 4.83 \times 10^{-12}$ & $2.52 \times 10^{-10} \pm 2.24 \times 10^{-12}$ & $512 \pm 1.49$ & $490 \pm 3.1$ & _- & _- \\
\hline & $15 / 23$ & $7.68 \times 10^{-10} \pm 1 \times 10^{-11}$ & $4.68 \times 10^{-10} \pm 1 \times 10^{-11}$ & $506 \pm 1.35$ & $581 \pm 1.74$ & _- & - \\
\hline $\begin{array}{l}\text { Dense Film } \\
300 \mu \mathrm{m} \\
25{ }^{\circ} \mathrm{C}\end{array}$ & 10 & $3.5 \times 10^{-8} \pm 7 \times 10^{-9}$ & & $1459 \pm 154$ & & - & \\
\hline
\end{tabular}

anitial casting thickness of the film forming solution.

Drying temperature of the film forming solution.

c The ratio corresponds to weight percentages of CA and water. Weight percentages of Psb and acetone in the film forming solution are 2 and 60 , respectively.

d Weight percentage of Psb in the film forming solution is 2.

${ }^{\mathrm{e}}$ The difference in diffusivities of Psb determined with the porous surfaces of the multilayer film prepared from $10 \% \mathrm{CA}$ and dried at $25^{\circ} \mathrm{C}$ and $50{ }^{\circ} \mathrm{C}$ is not statistically significant (p $>0.05$ ).

${ }^{\mathrm{f}}$ The difference in partition coefficients of Psb determined with the porous and dense surfaces of the multilayer film prepared from $15 \% \mathrm{CA}$ and dried at $25{ }^{\circ} \mathrm{C}$ is not statistically significant ( $\mathrm{p}>0.05$ ).

$\mathrm{g}$ The difference in partition coefficients of Psb determined with the dense surfaces of the monolayer film prepared from $12.5 \% \mathrm{CA}$ and dried at $25^{\circ} \mathrm{C}$ and $50{ }^{\circ} \mathrm{C}$ is not statistically significant ( $\mathrm{p}>0.05$ ).

${ }^{\mathrm{h}}$ The difference in dissolution constants of Psb determined with the porous surface of the monolayer film prepared from $10 \% \mathrm{CA}$ and dried at $25^{\circ} \mathrm{C}$ and $50^{\circ} \mathrm{C}$ is not statistically significant $(p>0.05)$.

${ }^{\mathrm{i}}$ The difference in dissolution constants of Psb determined with the porous surface of the monolayer films prepared from $12.5 \% \mathrm{CA}$ and dried at $25{ }^{\circ} \mathrm{C}$ and $50{ }^{\circ} \mathrm{C}$ is not statistically significant $(p>0.05)$.

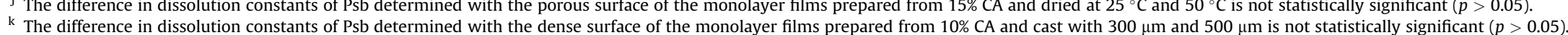

${ }^{1}$ The difference in dissolution constants of Psb determined with the dense surface of the monolayer films prepared from $15 \% \mathrm{CA}$ and dried at $25{ }^{\circ} \mathrm{C}$ and $50{ }^{\circ} \mathrm{C}$ is not statistically significant $(p>0.05$ ).

The difference in dissolution constants of Psb determined with the dense and porous surfaces of the monolayer film prepared from $10 \% \mathrm{CA}$ and dried at $50{ }^{\circ} \mathrm{C}$ is not statistically significant $(p>0.05)$.

The difference in dissolution constants of Psb determined with the porous surface of the monolayer film prepared from $10 \%$ CA and $12.5 \%$ CA and cast with $300 \mu \mathrm{m}$ is not statistically significant ( $p>0.05$ ).

${ }^{\circ}$ The difference in dissolution constants of Psb determined with the porous surface of the monolayer film prepared from $10 \% \mathrm{CA}$ and $12.5 \% \mathrm{CA}$ and cast with $500 \mu \mathrm{m}$ is not statistically significant $(p>0.05)$. 
monolayer films are higher compared with that in the multilayer films.

\section{Conclusions}

In this study, cellulose acetate based mono and multilayer films including potassium sorbate as an antimicrobial agent were prepared using the dry phase inversion technique. This technique allows production of novel food packaging materials with structures ranging from asymmetric and porous to dense by changing the initial casting composition, wet casting thickness and drying temperature. The porous structure initiated drying-induced crystal formation in the monolayer films. Psb release from these films was significantly prolonged since the fast initial burst in the release rate, controlled by Fickian diffusion, was followed by a decrease in the rate due to the slow dissolution of the crystals. It was found that the amount of Psb crystals and their size can be varied with the overall porosity and pore size of the films. Notably, however, this controlled and extended release was only observed in the monolayer films prepared by the dry phase inversion technique, whereas, the release rate from the dense monolayer film prepared by the traditional solvent evaporation technique was regulated only by the diffusion of Psb. Consequently, equilibrium was reached in a shorter time. All these results indicate that the dry phase inversion technique can be used for inducing Psb crystal formation and, hence, for achieving the controlled and extended release of Psb by having the release rate at longer times being controlled by the dissolution of the Psb crystals. Similar to the results for the dense monolayer film, the release rate through the multilayer films was regulated only by the diffusion of Psb.

In conclusion, the results suggest that the films prepared in this study may be used as novel, controlled release food packaging materials. However, further studies are needed to measure their antimicrobial activities on selected microorganisms and on real food surfaces. To the best of our knowledge, this is the first study which has shown drying-induced Psb crystal formation in food packaging materials and the preparation of crystal dissolutiondiffusion controlled release systems for food packaging applications.

\section{References}

Appendini, P., \& Hotchkiss, J. H. (2002). Review of antimicrobial food packaging Innovative Food Science and Emerging Technologies, 3, 113-126.

Berens, A. R., \& Hopfenberg, H. B. (1978). Diffusion and relaxation in glassy polymer powders: 2 separation of the diffusion and relaxation parameters. Polymer, 19, $489-496$.

Buonocore, G. G., Conte, A., Corbo, M. R., Sinigaglia, M., \& Del Nobile, M. A. (2005) Mono and multilayer active films containing lysozyme as antimcirobial agent. Innovative Food Science and Emerging Technologies, 6, 459-464.

Buonocore, G. G., Del Nobile, M. A., Panizza, A., Bove, S., Battaglia, G., \& Nicolas, L (2003). Modeling the lysozyme release kinetics from antimicrobial films intended for food packaging applications. Journal of Food Science, 68, 1365-1370.

Buonocore, G. G., Del Nobile, M. A., Panizza, A., Corbo, M. R., \& Nicolais, L. (2003). A general approach to describe the antimicrobial agent release from highly swellable films intended for food packaging applications. Journal of Controlled Release, 90, 97-107.

Buonocore, G. G., Sinigaglia, M., Corbo, M. R., Bevilacqua, A., La Notte, E., \& Del Nobile, M. A. (2004). Controlled release of antimicrobial compounds from highly swellable polymers. Journal of Food Protection, 67, 1190-1194.

Choi, J. H., Choi, W. Y., Cha, D. S., Chinnan, M. J., Park, H. J., Lee, D. S., et al. (2005). Diffusivity of potassium sorbate in k-carrageenan based antimicrobial film. LWT-Food Science and Technology, 38, 417-423.

Crank, J. (1975). The mathematics of diffusion ( $2^{\text {nd }}$ ed.). New York: Oxford University Press.

Fama, L., Flores, S. K., Gerschenson, L., \& Goyanes, S. (2006). Physical characterization of cassava starch biofilms with special reference to dynamic mechanical properties at low temperatures. Carbohydrate Polymers, 66, 8-15.

Flores, S., Haedo, A. S., Campos, C., \& Gerschenson, L. (2007). Antimicrobial performance of potassium sorbate supported in tapioca starch edible films. European Food Research and Technology, 225, 375-384.

Gemili, S., Yemenicioğlu, A., \& Altınkaya, S. A. (2010). Development of antioxidant food packaging materials with controlled release properties. Journal of Food Engineering, 96, 325-332.

Gemili, S., Yemenicioglu, A., \& Altınkaya, S. A. (2009). Development of cellulose acetate based antimicrobial food packaging materials for controlled release of lysozyme. Journal of Food Engineering, 90, 453-462.

Guzey, D., \& McClements, D. J. (2006). Formation, stability and properties of multilayer emulsions for application in the food industry. Advances in Colloid and Interface Science, 128-130, 227-248.

Han, J., Castell-Perez, M. E., \& Moreira, R. G. (2008). Effect of food characteristics, storage conditions, and electron beam irradiation on active agent release from polyamide-coated LDPE films. Journal of Food Science, 73, 37-43.

Han, J. H., \& Floros, J. D. (1997). Casting antimicrobial packaging films and measuring their physical properties and antimicrobial activity. Journal of Plastic Film and Sheeting, 13, 287-298.

Han, J. H., \& Floros, J. D. (1998). Simulating diffusion model and determining diffusivity of potassium sorbate through plastics to develop antimicrobial packaging films. Journal of Food Processing and Preservation, 22, 107-122.

Iconomopoulou, S. M., \& Voyiatzis, G. A. (2005). The effect of the molecular orientation on the release of antimicrobial substances from uniaxially drawn polymer matrixes. Journal of Controlled Release, 103, 451-464.

Limjaroen, P., Ryser, E., Lockhart, H., \& Harte, B. (2003). Development of a food packaging coating material with antimicrobial properties. Journal of Plastic Film and Sheeting, 19, 95-109.

Mastromatteo, M., Barbuzzi, G., Conte, A., \& Del Nobile, M. A. (2009). Controlled release of thymol from zein based film. Innovative Food Science and Emerging Technologies, 10, 222-227.

Min, S., \& Krochta, J. M. (2005). Inhibition of penicillium commune by edible whey protein films incorporating lactoferrin, lactoferrin hydrosylate and lactoperoxidase systems. Journal of Food Science, 70, 87-94.

Ozdemir, M., \& Floros, J.D.(2001). Analysis and modeling of potassium sorbate diffusion through edible whey protein films. Journal of Food Engineering, 47, 149-155.

Ozdemir, M., \& Floros, J. D. (2003). Film composition effects on diffusion of potassium sorbate through whey protein films. Journal of Food Science, 68, 511-516.

Valencia-Chamorro, S. A , Palou, L, Del Rio, M. A \& Perez-Gago, M. B. (2008) Inhibition of penicillium digitatum and penicillium italicum by hydroxypropyl methylcellulose-lipid edible composite films containin food additives with antifungal properties. Journal of Agricultural and Food Chemistry, 56, 11270-11278.

Vartiainen, J., Skytta, E., Enqvist, J., \& Ahvenainen, R. (2003). Properties of antimicrobial plastics containing traditional food preservatives. Packaging Technology and Science, 16, 223-229.

Ye, M., Neetoo, H., \& Chen, H. (2008). Effectiveness of chitosan-coated plastic films incorporating antimicrobials in inhibition of listeria monocytogenes on coldsmoked salmon. International Journal of Food Microbiology, 127, 235-240. 